\title{
EGFR Exon 19 Deletion Mutation
}

National Cancer Institute

\section{Source}

National Cancer Institute. EGFR Exon 19 Deletion Mutation. NCI Thesaurus. Code C126892.

In-frame deletions occurring within the exon 19-encoded part of the epidermal growth factor receptor (EGFR) kinase domain. These mutations are commonly seen in non-small cell lung cancer and are associated with increased sensitivity to EGFR tyrosine kinase inhibitors. 